\title{
5\% of Zion: Evaluating the potential for probability-split trades in professional sports
}

\author{
Dawson Brown* \\ Christopher Newport University, Economics, Sheridan, MT, USA
}

Received 18 May 2020

Accepted 20 October 2021

Pre-press 23 February 2022

Published 8 July 2022

\begin{abstract}
In this paper, I propose and evaluate a novel extension of the analytics revolution in professional sports: probabilitysplit trades. Under this plan, teams could trade probability shares in draft assets held. For example, a team like the New York Knicks could trade their first and second round picks for a $5 \%$ chance of winning the 1 st overall pick. In the last two decades, the analytics revolution has transformed professional sports. General managers, coaches, and even players leverage the underlying math to gain any sort of competitive advantage, while major sports leagues view the analytics revolution with passive glee, as their potential viewer segments continue to expand. This paper is an extension of that revolution, outlining the details, feasibility, and potential benefits of a novel plan with the potential to increase exchange efficiency, boost revenue and sustain league growth in the NFL and NBA.
\end{abstract}

Keywords: Management, risk, sports analytics, mutual exchange, trades

\section{Introduction}

In the last two decades, the analytics revolution has transformed professional sports (Fry \& Ohlmann, 2012; Davenport, 2014). Moneyball captivated the attention of fans and owners alike. The Sloan Conference has become a cultural phenomenon, bringing media and management together to revel in achievements and rejoice about the future of quantitative sports analysis. Each year, the models become more accurate, the hiring potential for young sports-oriented quants becomes greater, and interest in analytics among fans becomes more mainstream. General managers, coaches, and even players leverage the underlying math to gain any sort of competitive advantage, while major sports leagues view the analytics revolution with passive glee, as their potential viewer segments continue to expand. Players are more efficient, coaches are more informed,

\footnotetext{
${ }^{*}$ Corresponding author: Dawson Brown, Christopher Newport University, Economics, P.O. Box 872, 59749, Sheridan, MT, USA. Tel.: +1 4065810961; E-mail: dawson.brown.15@cnu.edu.
}

general managers are smarter, and leagues are richer - what could be wrong with this analytics revolution. In my estimation, just one thing; it does not quite extend far enough.

In this paper, I propose and evaluate a novel extension of the analytics revolution in professional sports: probability-split trades. Under this plan, teams could trade probability shares in draft assets held. For example, in the 2020 NFL draft, Joe Burrow was selected by the Cincinnati Bengals with the first overall pick. Under this proposal, the New England Patriots, who with the departure of Tom Brady seem to be in the market for a quarterback, could trade their draft assets for a $10 \%$ probability share of the first overall pick. In other words, the New England Patriots could do something like trade their fifth, sixth, and seventh round picks for a $10 \%$ chance of winning the 1 st overall pick, if of course, the Cincinnati Bengals agreed to the terms.

Before each draft, an event, much like the NBA lottery, would be held, at which the winner of the asset is determined via ping pong drawing. In this 
case, 90 ping pong balls would be pro-Cincinnati and 10 would be pro-Patriot. If a Cincinnati ping pong ball is pulled, the Bengals retain their first overall pick and also get the fifth, sixth, and seventh round picks of the Patriots. If the Patriots ping pong ball is pulled, the Patriots get the first overall pick, but still lose their fifth, sixth, and seventh round picks to the Bengals (because they traded a $100 \%$ probability share in these picks.

This paper outlines the details, feasibility, and potential benefits of such a plan in major professional sports, focusing on implications in the NBA and NFL specifically, due to the increased draft interest annually in these two leagues.

\section{Barter and the existing trade model}

Primarily, teams conduct trades in major professional sports through barter style transactions (Marburger, 2009). Both teams mutually exchange assets, that in theory, make both teams better off. In certain circumstances, cash itself is included as part of the trade; however, usually only player and draft assets are exchanged in trades. This existing system of trades in American major professional sports is well-defined by a barter model of transactions in economics, whereby two independent parties exchange goods or services directly without the use of money. Time and time again in the economic literature, barter systems have been shown to be inefficient (Marburger, 2009). First, any transaction in a barter system depends on a 'double coincidence of wants' - both parties have to possess what the other party wants. In other words, for the New Orleans Saints to complete a trade with the Miami Dolphins, New Orleans has to possess something that Miami wants and Miami has to possess something that New Orleans wants, only then can a trade occur.

Another inefficiency in barter-based trade systems is the lack of common measure of value. In any economic system, money usually represents the value in a good or service; therefore, price quickly and easily expresses value to both parties. In a barter system, the value of two things cannot be easily compared to one another because of the absence of price. In sports, players and picks are traded with ill-defined value, adding to the inefficiency of the existing barter trade model.

Perhaps most significantly, the existing barter model suffers from what economists call the 'indivisibility of goods'. Suppose I have a chicken and want to sell it for a wrench. If the wrench salesman values his wrench at the value of two chickens, then no trade can occur, because the wrench salesman cannot sell me half of a wrench. That is the problem with the existing trade model in American professional sports. Suppose that in the 2019 NBA draft, the Portland Trailblazers wanted to trade up to select Zion Williamson, the first overall pick in the draft. If Portland/New Orleans both valued Portland's 25th overall pick as a $\$ 5,000,000$ asset and the number one overall pick as a $\$ 100,000,000$ asset - no trade could possibly mend that gap in value. Portland could work with existing assets, potentially trading players like CJ McCollum and Jusuf Nurkic to try to get to $\$ 100,000,000$ in tradable assets; however, you still can't trade half of a player, so the inefficiency of indivisibility likely remains.

With the inherent inefficiency of the barter-based trade system in American professional sports, it is important to remember that draft pick assets are not inherently indivisible goods, like wrenches. Whereas challenges like the 'double coincidence of wants' and a lack of 'common measure of value' are difficult to overcome in the existing barter model of trades, the problem of indivisibility of goods would be much easier to solve. If the Portland Trailblazers wanted to trade up to select Zion Williamson and both Portland and New Orleans valued Portland's 25th overall pick as a $\$ 5,000,000$ asset and the number one overall pick as a $\$ 100,000,000$ asset. Then, in equilibrium, Portland should be willing to trade their 25th overall pick in the 2019 NBA draft for a 5\% probability share of the number one overall pick in the draft. On the other end of the bargain, the New Orleans Pelicans would also be willing to trade a $5 \%$ probability share of the number one overall pick in exchange for the 25th overall pick, while still retaining a $95 \%$ share of the first overall pick. Of course, this model oversimplifies asset evaluation, assuming that both teams assess at the same value. In reality, it is likely that no two teams would value each asset, whether pick or player, exactly the same. That reality does not challenge the underlying validity of the proposal, but rather creates uncertainty and confers advantages to general managers that are more adept at asset evaluation and managing uncertainty.

\section{The Plan}

In the probability-split trades plan, teams would be able to specify the probability share of any traded 
draft asset. Teams would certainly still be allowed to trade draft assets with a $100 \%$ probability share. If the New Orleans Saints wanted to trade two 100\% probability second round draft picks for a $100 \%$ probability share of Tampa Bay's first round pick, both parties could certainly do that. Trades involving players could also include partial shares of draft picks; however, teams could not trade probability shares of players. The Washington Wizards could not trade a first round pick for a $2 \%$ chance at Lebron James in the offseason, the probability shares would be exclusively applied to draft assets in this proposal.

Before each draft, an event, much like the NBA lottery, would be held, at which the winner of the asset is determined via ping pong ball drawing or some other random drawing. The teams involved in each trade would receive their appropriate share of ping pong balls and then the commissioner of the league would draw which team wins the control of the asset. Post-drawing, the asset would be $100 \%$ controlled by the winning team.

\section{Revenue implications}

The probability-split trades has the potential to not only make trade more efficient in major sports leagues, but also generate revenue for the leagues themselves. In 2019, 4.43 million people watched the NBA draft lottery on ESPN (Lewis, 2019). That rating was higher than $75 \%$ of NBA playoff games and $95 \%$ of Stanley Cup playoff games. Clearly, there exists a large market for draft content, random chance, and the bouncing of ping pong balls. Additionally, this type of telecast would have the potential to gain even greater interest than the draft lottery, as more teams could potentially be included. Fans would probably want their favorite NBA team to trade a second round pick for a $5 \%$ chance of being able to draft Zion Williamson, I know I would. On the other end of the spectrum, New Orleans Pelicans fans might want to trade $50 \%$ of the Zion Williamson probability to compile 10 additional draft picks. These probability-based split trades add a layer to each decision made that creates widespread intrigue and allows each league to extend their reach in the media cycle during the offseason. Of course, the revenue creation potential of this type of event is entirely dependent on teams actually utilizing the probability split-trade system.

\section{Management implications}

The imposition of the probability-split trade plan would confer advantages to general managers and team decision-makers that are more adept at asset evaluation, working with statistics, and managing uncertainty. With that being said, the dawn of the analytics revolution in sports has already conferred benefits to team decision-makers with those unique skill sets. Calculating expected values based on probability and asset evaluation would not be tremendously difficult for the teams of quantitative analysts that most major professional sports teams employ today, the challenge would still mostly lie with asset evaluation in the first place, which plagued general managers before teams even owned computers.

General Managers and decision-makers would also have to manage the additional wrinkle of fan expectations. Fans and decision-makers likely have different levels of value assigned to a seventh round pick in the NFL. In theory, the split trade plan allows each team to gain a probability share of any high first round pick. That potential is relatively nonexistent for most teams in the NFL draft presently. With that new hope in mind, fan-team relations may face different challenges after the imposition of such a plan.

\section{League policy and potential challenges}

As currently constructed, the official NFL and NBA rules do not outlaw probability-share trades (NFL Football Operations, 2021; NBA PA, 2021). In theory, if teams were willing to set up a random chance drawing between the two parties, they could carry out these types of trades without a league-wide drawing event; however, a league-wide event would offer tremendous revenue potential for the league.

The largest potential challenge to the probabilitysplit trade plan is inertia. If team decision-makers are unwilling or unable to utilize split trades, then leagues will lose the potential revenue from a random drawing event. If events do not make leagues money, they will likely be scrapped. The recent analytics revolution provides optimism in regard to this challenge. Analytics-driven general managers with the guidance of quantitative analysis teams should be able to utilize the split trade system to drive the league towards a more efficient equilibrium, conferring benefits to the league at large.

Another potential challenge is the high volume of draft assets exchange on draft day itself. As cur- 
rently constructed, these assets would be viewed as current players and thus could not be subject to a probability-split trade eligible. In this case, it is probably most feasible for draft day trades to be $100 \%$ probability-share only. All pre-draft trades would still be probability split.

\section{Conclusions}

In light of the analytics revolution that has transformed professional sports, teams and leagues are increasingly evaluating quantitatively-driven ways to gain competitive advantages. The proposed probability-split trade system would both allow teams to compete in a more efficient system, but also allow leagues to boost revenue and sustain growth. Initially, decision-makers may be unwilling or unable to utilize the new system, but the analytics revolution has taught us that competition will drive the game towards a more efficient equilibrium. This proposal is the next step forward towards that equilibrium - an equilibrium in which any NBA team, even the New York Knicks, can buy-in for a 5\% chance to win Lebron James or Zion Williamson.

\section{References}

Davenport, T. H., 2014, What businesses can learn from sports analytics, MIT Sloan Management Review, 55(4), 10.

Fry, M. J. \& Ohlmann, J. W., 2012, Introduction to the special issue on analytics in sports, part I: General sports applications.

Lewis, J. 2019, NBA Draft Lottery Up Big to 16-Year High. Retrieved from https://www.sportsmediawatch. com/2019/05/nba-draft-lottery-ratings-high-espn/

Marburger, D. R. 2009, Why Do Player Trades Dominate Sales? Journal of Sports Economics, 10(4), 335-350.

NBA PA. 2021, Collective Bargaining Agreement. Retrieved from https://nbpa.com/cba

NFL Football Operations. 2021, The Rules of the Draft. Retrieved from https://operations.nfl.com/the-players/the-nfl-draft/therules-of-the-draft/ 